\title{
Orthomodular Lattices
}

\author{
Elżbieta Mądra \\ Institute of Mathematics \\ University of Białystok \\ Akademicka 2, 15-267 Białystok \\ Poland
}

\author{
Adam Grabowski \\ Institute of Mathematics \\ University of Białystok \\ Akademicka 2, 15-267 Białystok \\ Poland
}

Summary. The main result of the article is the solution to the problem of short axiomatizations of orthomodular ortholattices. Based on EQP/Otter results [10], we gave a set of three equations which is equivalent to the classical, much longer equational basis of such a class. Also the basic example of the lattice which is not orthomodular, i.e. benzene (or $B_{6}$ ) is defined in two settings - as a relational structure (poset) and as a lattice.

As a preliminary work, we present the proofs of the dependence of other axiomatizations of ortholattices. The formalization of the properties of orthomodular lattices follows [4].

MML identifier: ROBBINS4, version: 7.9.03 4.104.1021

The articles [6], [11], [13], [5], [2], [1], [3], [14], [12], [7], [8], and [9] provide the terminology and notation for this paper.

\section{Preliminaries}

Let $L$ be a lattice. One can verify that the lattice structure of $L$ is lattice-like. Next we state the proposition

(1) For all lattices $K, L$ such that the lattice structure of $K=$ the lattice structure of $L$ holds $\operatorname{Poset}(K)=\operatorname{Poset}(L)$.

Let us note that every non empty ortholattice structure which is trivial is also quasi-meet-absorbing.

One can check that every ortholattice is lower-bounded and every ortholattice is upper-bounded. 
In the sequel $L$ denotes an ortholattice and $a, b, c$ denote elements of $L$.

We now state three propositions:

(2) $a \sqcup a^{\mathrm{c}}=\top_{L}$ and $a \sqcap a^{\mathrm{c}}=\perp_{L}$.

(3) Let $L$ be a non empty ortholattice structure. Then $L$ is an ortholattice if and only if the following conditions are satisfied:

(i) for all elements $a, b, c$ of $L$ holds $a \sqcup b \sqcup c=\left(c^{\mathrm{c}} \sqcap b^{\mathrm{c}}\right)^{\mathrm{c}} \sqcup a$,

(ii) for all elements $a, b$ of $L$ holds $a=a \sqcap(a \sqcup b)$, and

(iii) for all elements $a, b$ of $L$ holds $a=a \sqcup\left(b \sqcap b^{\mathrm{c}}\right)$.

(4) Let $L$ be an involutive lattice-like non empty ortholattice structure. Then $L$ is de Morgan if and only if for all elements $a, b$ of $L$ such that $a \sqsubseteq b$ holds $b^{\mathrm{c}} \sqsubseteq a^{\mathrm{c}}$.

\section{Orthomodularity}

Let $L$ be a non empty ortholattice structure. We say that $L$ is orthomodular if and only if:

(Def. 1) For all elements $x, y$ of $L$ such that $x \sqsubseteq y$ holds $y=x \sqcup\left(x^{\mathrm{c}} \sqcap y\right)$.

Let us observe that there exists an ortholattice which is trivial, orthomodular, modular, and Boolean.

Next we state the proposition

(5) Every modular ortholattice is orthomodular.

An orthomodular lattice is an orthomodular ortholattice.

One can prove the following proposition

(6) Let $L$ be an orthomodular meet-absorbing join-absorbing join-associative meet-commutative non empty ortholattice structure and $x, y$ be elements of $L$. Then $x \sqcup\left(x^{\mathrm{c}} \sqcap(x \sqcup y)\right)=x \sqcup y$.

Let $L$ be a non empty ortholattice structure. We say that $L$ satisfies OM if and only if:

(Def. 2) For all elements $x, y$ of $L$ holds $x \sqcup\left(x^{\mathrm{c}} \sqcap(x \sqcup y)\right)=x \sqcup y$.

Let us observe that every meet-absorbing join-absorbing join-associative meet-commutative non empty ortholattice structure which satisfies OM is also orthomodular and every meet-absorbing join-absorbing join-associative meetcommutative non empty ortholattice structure which is orthomodular satisfies also OM.

Let us observe that every ortholattice which is modular is also orthomodular.

Let us mention that there exists an ortholattice which is quasi-joinassociative, quasi-meet-absorbing, de Morgan, and orthomodular. 


\section{Examples: The Benzene Ring}

The relational structure $B_{6}$ is defined by:

(Def. 3$) \quad B_{6}=\langle\{0,1,3 \backslash 1,2,3 \backslash 2,3\}, \subseteq\rangle$.

Let us note that $B_{6}$ is non empty and $B_{6}$ is reflexive, transitive, and antisymmetric.

Let us note that $B_{6}$ has l.u.b.'s and g.l.b.'s.

One can prove the following propositions:

(7) The carrier of $\mathbb{L}_{B_{6}}=\{0,1,3 \backslash 1,2,3 \backslash 2,3\}$.

(8) For every set $a$ such that $a \in$ the carrier of $\mathbb{L}_{B_{6}}$ holds $a \subseteq 3$.

The strict ortholattice structure Benzene is defined by the conditions (Def. 4).

(Def. 4)(i) The lattice structure of Benzene $=\mathbb{L}_{B_{6}}$, and

(ii) for every element $x$ of the carrier of Benzene and for every subset $y$ of 3 such that $x=y$ holds (the complement operation of Benzene) $(x)=y^{\mathrm{c}}$.

The following three propositions are true:

(9) The carrier of Benzene $=\{0,1,3 \backslash 1,2,3 \backslash 2,3\}$.

(10) The carrier of Benzene $\subseteq 2^{3}$.

(11) For every set $a$ such that $a \in$ the carrier of Benzene holds $a \subseteq\{0,1,2\}$.

Let us note that Benzene is non empty and Benzene is lattice-like.

The following propositions are true:

(12) Poset(the lattice structure of Benzene) $=B_{6}$.

(13) For all elements $a, b$ of $B_{6}$ and for all elements $x, y$ of Benzene such that $a=x$ and $b=y$ holds $a \leq b$ iff $x \sqsubseteq y$.

(14) For all elements $a, b$ of $B_{6}$ and for all elements $x, y$ of Benzene such that $a=x$ and $b=y$ holds $a \sqcup b=x \sqcup y$ and $a \sqcap b=x \sqcap y$.

(15) For all elements $a, b$ of $B_{6}$ such that $a=3 \backslash 1$ and $b=2$ holds $a \sqcup b=3$ and $a \sqcap b=0$.

(16) For all elements $a, b$ of $B_{6}$ such that $a=3 \backslash 2$ and $b=1$ holds $a \sqcup b=3$ and $a \sqcap b=0$.

(17) For all elements $a, b$ of $B_{6}$ such that $a=3 \backslash 1$ and $b=1$ holds $a \sqcup b=3$ and $a \sqcap b=0$.

(18) For all elements $a, b$ of $B_{6}$ such that $a=3 \backslash 2$ and $b=2$ holds $a \sqcup b=3$ and $a \sqcap b=0$.

(19) For all elements $a, b$ of Benzene such that $a=3 \backslash 1$ and $b=2$ holds $a \sqcup b=3$ and $a \sqcap b=0$.

(20) For all elements $a, b$ of Benzene such that $a=3 \backslash 2$ and $b=1$ holds $a \sqcup b=3$. 
(21) For all elements $a, b$ of Benzene such that $a=3 \backslash 1$ and $b=1$ holds $a \sqcup b=3$.

(22) For all elements $a, b$ of Benzene such that $a=3 \backslash 2$ and $b=2$ holds $a \sqcup b=3$.

(23) Let $a$ be an element of Benzene. Then

(i) if $a=0$, then $a^{\mathrm{c}}=3$,

(ii) if $a=3$, then $a^{\mathrm{c}}=0$,

(iii) if $a=1$, then $a^{\mathrm{c}}=3 \backslash 1$,

(iv) if $a=3 \backslash 1$, then $a^{\mathrm{c}}=1$,

(v) if $a=2$, then $a^{\mathrm{c}}=3 \backslash 2$, and

(vi) if $a=3 \backslash 2$, then $a^{\mathrm{c}}=2$.

(24) For all elements $a, b$ of Benzene holds $a \sqsubseteq b$ iff $a \subseteq b$.

(25) For all elements $a, x$ of Benzene such that $a=0$ holds $a \sqcap x=a$.

(26) For all elements $a, x$ of Benzene such that $a=0$ holds $a \sqcup x=x$.

(27) For all elements $a, x$ of Benzene such that $a=3$ holds $a \sqcup x=a$.

One can check that Benzene is lower-bounded and Benzene is upperbounded.

We now state two propositions:

(28) $T_{\text {Benzene }}=3$.

(29) $\perp_{\text {Benzene }}=0$.

Let us note that Benzene is involutive and de Morgan and has top and Benzene is non orthomodular.

\section{Orthogonality}

Let $L$ be an ortholattice and let $a, b$ be elements of $L$. We say that $a, b$ are orthogonal if and only if:

(Def. 5) $a \sqsubseteq b^{\mathrm{c}}$.

Let $L$ be an ortholattice and let $a, b$ be elements of $L$. We introduce $a \perp b$ as a synonym of $a, b$ are orthogonal.

Next we state the proposition

(30) $a \perp a$ iff $a=\perp_{L}$.

Let $L$ be an ortholattice and let $a, b$ be elements of $L$. Let us note that the predicate $a, b$ are orthogonal is symmetric.

The following proposition is true

(31) If $a \perp b$ and $a \perp c$, then $a \perp b \sqcap c$ and $a \perp b \sqcup c$. 


\section{Orthomodularity Conditions}

One can prove the following propositions:

(32) $L$ is orthomodular iff for all elements $a, b$ of $L$ such that $b^{\mathrm{c}} \sqsubseteq a$ and $a \sqcap b=\perp_{L}$ holds $a=b^{\mathrm{c}}$.

(33) $L$ is orthomodular iff for all elements $a, b$ of $L$ such that $a \perp b$ and $a \sqcup b=\top_{L}$ holds $a=b^{\mathrm{c}}$.

(34) $L$ is orthomodular iff for all elements $a, b$ of $L$ such that $b \sqsubseteq a$ holds $a \sqcap\left(a^{\mathrm{c}} \sqcup b\right)=b$.

(35) $L$ is orthomodular iff for all $a, b$ holds $a \sqcap\left(a^{\mathrm{c}} \sqcup(a \sqcap b)\right)=a \sqcap b$.

(36) $L$ is orthomodular iff for all elements $a, b$ of $L$ holds $a \sqcup b=((a \sqcup b) \sqcap$ a) $\sqcup\left((a \sqcup b) \sqcap a^{\mathrm{c}}\right)$.

(37) $L$ is orthomodular iff for all $a, b$ such that $a \sqsubseteq b$ holds $(a \sqcup b) \sqcap\left(b \sqcup a^{\mathrm{c}}\right)=$ $(a \sqcap b) \sqcup\left(b \sqcap a^{\mathrm{c}}\right)$.

(38) Let $L$ be a non empty ortholattice structure. Then $L$ is an orthomodular lattice if and only if the following conditions are satisfied:

(i) for all elements $a, b, c$ of $L$ holds $a \sqcup b \sqcup c=\left(c^{\mathrm{c}} \sqcap b^{\mathrm{c}}\right)^{\mathrm{c}} \sqcup a$,

(ii) for all elements $a, b, c$ of $L$ holds $a \sqcup b=((a \sqcup b) \sqcap(a \sqcup c)) \sqcup\left((a \sqcup b) \sqcap a^{\mathrm{c}}\right)$, and

(iii) for all elements $a, b$ of $L$ holds $a=a \sqcup\left(b \sqcap b^{\mathrm{c}}\right)$.

One can verify that every quasi-join-associative quasi-meet-absorbing de Morgan orthomodular lattice-like non empty ortholattice structure has top.

Next we state the proposition

(39) Let $L$ be a non empty ortholattice structure. Then $L$ is an orthomodular lattice if and only if $L$ is quasi-join-associative, quasi-meet-absorbing, and de Morgan and satisfies OM.

\section{REFERENCES}

[1] Grzegorz Bancerek. Cardinal numbers. Formalized Mathematics, 1(2):377-382, 1990.

[2] Grzegorz Bancerek. The ordinal numbers. Formalized Mathematics, 1(1):91-96, 1990.

[3] Grzegorz Bancerek. Complete lattices. Formalized Mathematics, 2(5):719-725, 1991.

[4] Ladislav Beran. Orthomodular Lattices. Algebraic Approach. Academiai Kiado, 1984.

[5] Czesław Byliński. Functions and their basic properties. Formalized Mathematics, 1(1):5565, 1990.

[6] Czesław Byliński. Some basic properties of sets. Formalized Mathematics, 1(1):47-53, 1990.

[7] Adam Grabowski. Robbins algebras vs. Boolean algebras. Formalized Mathematics, $9(4): 681-690,2001$.

[8] Adam Grabowski and Robert Milewski. Boolean posets, posets under inclusion and products of relational structures. Formalized Mathematics, 6(1):117-121, 1997.

[9] Adam Grabowski and Markus Moschner. Formalization of ortholattices via orthoposets. Formalized Mathematics, 13(1):189-197, 2005.

[10] W. McCune, R. Padmanabhan, M. A. Rose, and R. Veroff. Automated discovery of single axioms for ortholattices. Algebra Universalis, 52(4):541-549, 2005.

[11] Andrzej Trybulec. Enumerated sets. Formalized Mathematics, 1(1):25-34, 1990. 
[12] Wojciech A. Trybulec and Grzegorz Bancerek. Kuratowski - Zorn lemma. Formalized Mathematics, 1(2):387-393, 1990.

[13] Zinaida Trybulec. Properties of subsets. Formalized Mathematics, 1(1):67-71, 1990.

[14] Stanisław Żukowski. Introduction to lattice theory. Formalized Mathematics, 1(1):215222, 1990.

Received June 27, 2008 